\title{
The Weight Distributions of a Class of Cyclic Codes with Three Nonzeros over $\mathbb{F}_{3}$
}

\author{
Xiaogang Liu and Yuan Luo \\ Computer Science and Engineering Department, Shanghai Jiao Tong University, Shanghai 200240, China \\ Correspondence should be addressed to Yuan Luo; yuanluo@sjtu.edu.cn
}

Received 6 April 2014; Accepted 22 June 2014; Published 9 July 2014

Academic Editor: Xing Jin

Copyright (C) 2014 X. Liu and Y. Luo. This is an open access article distributed under the Creative Commons Attribution License, which permits unrestricted use, distribution, and reproduction in any medium, provided the original work is properly cited.

Cyclic codes have efficient encoding and decoding algorithms. The decoding error probability and the undetected error probability are usually bounded by or given from the weight distributions of the codes. Most researches are about the determination of the weight distributions of cyclic codes with few nonzeros, by using quadratic forms and exponential sums but limited to low moments. In this paper, we focus on the application of higher moments of the exponential sums to determine the weight distributions of a class of ternary cyclic codes with three nonzeros, combining with not only quadratic forms but also MacWilliams' identities. Another application of this paper is to emphasize the computer algebra system Magma for the investigation of the higher moments. In the end, the result is verified by one example using Matlab.

\section{Introduction}

Cyclic codes have a lot of applications in communication system, storage system, and computers, and they have been studied for a long time $[1,2]$. The decoding error probability and the undetected error probability are closely related to the weight distributions, for example, permutation decoding, majority decoding, locator decoding, decoding from the covering polynomials, and so on [3-6]. In general the weight distributions are complicated [7] and difficult to be determined. In fact, as shown in $[8,9]$, the problem of computing weight distribution of a cyclic code is connected with the evaluation of certain exponential sums, which are generally hard to be determined explicitly. For more researches, refer to [10-13] for the irreducible case, [14-17] for the reducible case, and [18-21] for recent studies. For related problems in the binary case with two nonzeros especially, refer to [22-24].

In this paper, we focus on the application of higher moments of the exponential sums to determine the weight distributions of a class of ternary cyclic codes with three nonzeros, combining with not only quadratic forms but also MacWilliams' identities, with the help of the computer algebra system Magma.
Let $p$ be a prime. A linear $[n, k, d ; p]$ code is a $k$ dimensional subspace of $\mathbb{F}_{p}^{n}$ with minimum (Hamming) distance $d$. An $[n, k]$ linear code $\mathscr{C}$ over $\mathbb{F}_{p}$ is called cyclic if $\left(c_{0}, c_{1}, \ldots, c_{n-1}\right) \in \mathscr{C}$ implies that $\left(c_{n-1}, c_{0}, c_{1}, \ldots, c_{n-2}\right) \in$ $\mathscr{C}$ where $\operatorname{gcd}(n, p)=1$. By identifying the vector $\left(a_{0}, a_{1}, \ldots, a_{n-1}\right) \in \mathbb{F}_{p}^{n}$ with

$$
a_{0}+a_{1} x+\cdots+a_{n-1} x^{n-1} \in \frac{\mathbb{F}_{p}[x]}{\left(x^{n}-1\right)}
$$

any linear code $\mathscr{C}$ of length $n$ over $\mathbb{F}_{p}$ represents a subset of $\mathbb{F}_{p}[x] /\left(x^{n}-1\right)$ which is a principle ideal domain. The fact that the code is cyclic is equivalent to the fact that the subset is an ideal. The unique monic polynomial $g(x)$ of minimum degree in this subset is the generating polynomial of $\mathscr{C}$, and it is a factor of $x^{n}-1$. When the ideal does not contain any smaller nonzero ideal, the corresponding cyclic code $\mathscr{C}$ is called a minimal or an irreducible code. For any $v=\left(c_{0}, c_{1}, \ldots, c_{n-1}\right) \in$ $\mathscr{C}$, the weight of $v$ is $w t(v)=\#\left\{c_{i} \neq 0, i=0,1, \ldots, n-1\right\}$.

The weight enumerator of a code $\mathscr{C}$ is defined by

$$
1+A_{1} x+A_{2} x^{2}+\cdots+A_{n} x^{n},
$$

where $A_{i}$ denotes the number of codewords with Hamming weight $i$. The sequence $1, A_{1}, \ldots, A_{n}$ is called the weight 
distribution of the code, which is an important parameter of a linear block code.

Assume that $p=3$ and $q=p^{m}$ for an even integer $m$. Let $\pi$ be a primitive element of $\mathbb{F}_{q}$. In this paper, Section 2 presents the basic notations and preliminaries about cyclic codes. Section 3 determines the weight distributions of a class of cyclic codes over $\mathbb{F}_{3}$ with nonzeros $\pi^{-2}, \pi^{-4}$, and $\pi^{-10}$, and they are verified by using Matlab. Note that the length of the cyclic code is $l=q-1=3^{m}-1$. Final conclusion is in Section 4. This paper is the counterpart of our other result in [19].

\section{Preliminaries}

In this section, relevant knowledge from finite fields [25] is presented for our study of cyclic codes. It is about the calculations of exponential sums, the sizes of cyclotomic cosets, and the ranks of certain quadratic forms. First, some known properties about the codeword weight are listed.

Let $p$ be an odd prime and let $m$ be a positive integer and $\pi$ is a primitive element of $\mathbb{F}_{q}$. Assume that the cyclic code $\mathscr{C}$ over $\mathbb{F}_{p}$ has length $l=q-1=p^{m}-1$ and nonconjugate nonzeros $\pi^{-s_{\lambda}}$, where $1 \leq s_{\lambda} \leq q-2(1 \leq \lambda \leq \iota)$. Then the codewords in $\mathscr{C}$ can be expressed by

$$
c\left(\alpha_{1}, \ldots, \alpha_{\iota}\right)=\left(c_{0}, c_{1}, \ldots, c_{l-1}\right) \quad\left(\alpha_{1}, \ldots, \alpha_{\iota} \in \mathbb{F}_{q}\right),
$$

where $c_{i}=\sum_{\lambda=1}^{l} \operatorname{Tr}\left(\alpha_{\lambda} \pi^{i s_{\lambda}}\right)(0 \leq i \leq l-1)$ and $\operatorname{Tr}: \mathbb{F}_{q} \rightarrow \mathbb{F}_{p}$ is the trace mapping from $\mathbb{F}_{q}$ to $\mathbb{F}_{p}$. Therefore the Hamming weight of the codeword $c=c\left(\alpha_{1}, \ldots, \alpha_{\iota}\right)$ is

$$
\begin{aligned}
w_{H}(c) & =\#\left\{i \mid 0 \leq i \leq l-1, c_{i} \neq 0\right\} \\
& =l-\frac{l}{p}-\frac{1}{p} \sum_{a=1}^{p-1} \sum_{x \in \mathbb{F}_{q}^{*}} \zeta_{p}^{\operatorname{Tr}(a f(x))} \\
& =p^{m-1}(p-1)-\frac{1}{p} \sum_{a=1}^{p-1} S\left(a \alpha_{1}, \ldots, a \alpha_{\iota}\right) \\
& =p^{m-1}(p-1)-\frac{1}{p} R\left(\alpha_{1}, \ldots, \alpha_{\iota}\right),
\end{aligned}
$$

where $\zeta_{p}=e^{2 \pi i / p}$ ( $i$ is imaginary unit), $f(x)=\alpha_{1} x^{s_{1}}+\alpha_{2} x^{s_{2}}+$ $\cdots+\alpha_{\iota} x^{s_{\iota}} \in \mathbb{F}_{q}[x], \mathbb{F}_{q}^{*}=\mathbb{F}_{q} \backslash\{0\}$,

$$
S\left(\alpha_{1}, \ldots, \alpha_{\iota}\right)=\sum_{x \in \mathbb{F}_{q}} \zeta_{p}^{\operatorname{Tr}\left(\alpha_{1} x^{s_{1}}+\cdots+\alpha_{\iota} x^{s_{l}}\right)}
$$

and $R\left(\alpha_{1}, \ldots, \alpha_{l}\right)=\sum_{a=1}^{p-1} S\left(a \alpha_{1}, \ldots, a \alpha_{\iota}\right)$.

For general functions of the form $f_{\alpha, \ldots, \gamma}(x)=\alpha x^{p^{i}+1}+\cdots+$ $\gamma x^{p^{j}+1}$ where $0 \leq i, \ldots, j \leq\lfloor m / 2\rfloor$, there are quadratic forms $F_{\alpha, \ldots, \gamma}(X)$ and corresponding symmetric matrices $H_{\alpha, \ldots, \gamma}$ satisfying that $F_{\alpha, \ldots, \gamma}(X)=X H_{\alpha, \ldots, \gamma} X^{T}=\operatorname{Tr}\left(f_{\alpha, \ldots, \gamma}(x)\right)$. It is known that there exists $M_{\alpha, \ldots, \gamma} \in \mathrm{GL}_{m}\left(\mathbb{F}_{p}\right)$ such that

$$
H_{\alpha, \ldots, \gamma}^{\prime}=M_{\alpha, \ldots, \gamma} H_{\alpha, \ldots, \gamma} M_{\alpha, \ldots, \gamma}^{T}=\operatorname{diag}\left(a_{1}, \ldots, a_{r_{\alpha, \ldots, \gamma},}, 0, \ldots, 0\right),
$$

where $a_{i} \in \mathbb{F}_{p}^{*}\left(1 \leq i \leq r_{\alpha, \ldots, \gamma}\right)$ and $r_{\alpha, \ldots, \gamma}=\operatorname{rank} H_{\alpha, \ldots, \gamma}$. Let $\Delta=a_{1} \cdots a_{r_{\alpha, \ldots, \gamma}}\left(\right.$ set $\Delta=1$ for $\left.r_{\alpha, \ldots, \gamma}=0\right)$.

Lemma 1 (Lemma 1, [15]). (i) For the quadratic form $F(X)=$ $X H X^{T}$,

$$
\sum_{X \in \mathbb{F}_{p}^{m}} \zeta_{p}^{F(X)}= \begin{cases}\left(\frac{\Delta}{p}\right) p^{m-r / 2} & \text { if } p \equiv 1(\bmod 4), \\ i^{r}\left(\frac{\Delta}{p}\right) p^{m-r / 2} & \text { if } p \equiv 3(\bmod 4) .\end{cases}
$$

(ii) For $A=\left(a_{1}, \ldots, a_{m}\right) \in \mathbb{F}_{p}^{m}$, if $2 Y H+A=0$ has solution $Y=B \in \mathbb{F}_{p}^{m}$, then

$$
\sum_{X \in \mathbb{F}_{p}^{m}} \zeta_{p}^{F(X)+A X^{T}}=\zeta_{p}^{c} \sum_{X \in \mathbb{F}_{p}^{m}} \zeta_{p}^{F(X)} \quad \text { where } c=\frac{1}{2} A B^{T} \in \mathbb{F}_{p}
$$

Otherwise $\sum_{X \in \mathbb{F}_{p}^{m}} \zeta_{p}^{F(X)+A X^{T}}=0$.

Here $(\Delta / p)$ denotes the Legendre symbol.

References [26, Lemma 9] and [19, Lemma 3] are about the calculations of exponential sums that will be needed in the sequel. For more details, refer to [15].

The cyclotomic coset containing $s$ is defined to be

$$
\mathscr{D}_{s}=\left\{s, s p, s p^{2}, \ldots, s p^{m_{s}-1}\right\}
$$

where $m_{s}$ is the smallest positive integer such that $p^{m_{s}} \cdot s \equiv$ $s\left(\bmod p^{m}-1\right)$. Reference [26, Lemma 10] is about the size of cyclotomic coset; also refer to [27] for the binary case.

For $f_{d}^{\prime}(x)=\alpha_{0} x^{2}+\alpha_{1} x^{p+1}+\cdots+\alpha_{d} x^{p^{d}+1}$ with corresponding quadratic form $F_{d}^{\prime}(X)=\operatorname{Tr}\left(f_{d}^{\prime}(x)\right)=X H_{d}^{\prime} X^{T}$, where $\left(\alpha_{0}, \alpha_{1}, \ldots, \alpha_{d}\right) \in \mathbb{F}_{q}^{d+1} \backslash\{(0,0, \ldots, 0)\}$. Reference [19, Corollary 1] is about its rank for a special case.

Note that in Section 3, a nonzero solution of an equation system means that all the variable values are nonzero.

\section{Main Result}

In this section, the main result of this paper is obtained; that is, the weight distribution of the cyclic code $C$ with nonzeros $\pi^{-2}, \pi^{-(p+1)}$ and $\pi^{-\left(p^{2}+1\right)}$ for the case $m$ is even; here $p=3$. For this, the first five moments of exponential sum $S(\alpha, \beta, \gamma)$ are computed in Sections 3.1, 3.2, and 3.3, and the MacWlliams' identities are calculated in Section 3.4.

3.1. The First Three Moments of $S(\alpha, \beta, \gamma)$. For an odd prime $p$ and even integer $m$, this subsection calculates the first three moments of the exponential sum $S(\alpha, \beta, \gamma)(5)$; see Lemma 2 and its other form Lemma 3.

Lemma 2 (see [19]). Let $p$ be an odd prime satisfying $p \equiv$ $3 \bmod 4$ and $q=p^{m}$. Then there are the following results about the exponential sum $S(\alpha, \beta, \gamma)$ (5) corresponding to $f_{2}^{\prime}(x)=$ $\alpha x^{2}+\beta x^{p+1}+\gamma x^{p^{2}+1}:$
(i) $\sum_{\alpha, \beta, \gamma \in \mathbb{F}_{q}} S(\alpha, \beta, \gamma)=p^{3 m}$, 
(ii) $\sum_{\alpha, \beta, \gamma \in \mathbb{F}_{q}} S(\alpha, \beta, \gamma)^{2}=p^{3 m}$,

(iii) $\sum_{\alpha, \beta, \gamma \in \mathbb{F}_{q}} S(\alpha, \beta, \gamma)^{3}=\left((p+1)\left(p^{m}-1\right)+1\right) p^{3 m}$.

Corresponding to Lemma 1 and [19, Corollary 1], we introduce the following notations for convenience. Let

$$
N_{\varepsilon, j}=\left\{(\alpha, \beta, \gamma) \in \mathbb{F}_{q}^{3} \backslash\{(0,0,0)\} \mid S(\alpha, \beta, \gamma)=\varepsilon p^{(m+j) / 2}\right\},
$$

where $\varepsilon= \pm 1$ and $j=0,2,4$. Denote $n_{\varepsilon, j}=\left|N_{\varepsilon, j}\right|$. Let

$$
N_{\varepsilon, j}=\left\{(\alpha, \beta, \gamma) \in \mathbb{F}_{q}^{3} \backslash\{(0,0,0)\} \mid S(\alpha, \beta, \gamma)=\varepsilon i p^{(m+j) / 2}\right\}
$$

for $j=1,3$, where $i$ is the imaginary unit. By [19, Lemma 3], set $n_{j}=n_{\varepsilon, j}=\left|N_{\varepsilon, j}\right|$ for $j=1,3$, since $m-j$ is odd. Using those, Lemma 2 can be restated in Lemma 3 when $m$ is even.

Lemma 3. Let $p$ be an odd prime satisfying $p \equiv 3 \bmod 4$, and $q=p^{m}$, where $m$ is an even integer. Then the notations defined in (10) and (11) satisfy

$$
\begin{aligned}
& 2\left(n_{1}+n_{3}\right)+n_{-1,0}+n_{1,0}+n_{-1,2}+n_{1,2}+n_{1,4}+n_{-1,4} \\
& \quad=p^{3 m}-1, \\
& n_{1,0}-n_{-1,0}+p\left(n_{1,2}-n_{-1,2}\right)+p^{2}\left(n_{1,4}-n_{-1,4}\right) \\
& \quad=p^{m / 2}\left(p^{2 m}-1\right), \\
& -2\left(p n_{1}+p^{3} n_{3}\right)+n_{1,0}+n_{-1,0}+p^{2}\left(n_{1,2}+n_{-1,2}\right) \\
& \quad+p^{4}\left(n_{1,4}+n_{-1,4}\right)=p^{m}\left(p^{m}-1\right), \\
& n_{1,0}-n_{-1,0}+p^{3}\left(n_{1,2}-n_{-1,2}\right)+p^{6}\left(n_{1,4}-n_{-1,4}\right) \\
& \quad=(p+1) p^{3 m / 2}\left(p^{m}-1\right) .
\end{aligned}
$$

Proof. Substituting the symbols of (10) and (11) to Lemma 2

$$
\begin{aligned}
& 2\left(n_{1}+n_{3}\right)+n_{-1,0}+n_{1,0}+n_{-1,2}+n_{1,2}+n_{1,4}+n_{-1,4} \\
& \quad=p^{3 m}-1,
\end{aligned}
$$

$$
\begin{aligned}
\sum_{\alpha, \beta, \gamma \in \mathbb{F}_{q}} S(\alpha, \beta, \gamma) & \\
= & p^{m / 2}\left(n_{1,0}-n_{-1,0}\right)+i p^{(m+1) / 2}\left(n_{1,1}-n_{-1,1}\right) \\
& +p^{(m+2) / 2}\left(n_{1,2}-n_{-1,2}\right)+i p^{(m+3) / 2}\left(n_{1,3}-n_{-1,3}\right) \\
& +p^{(m+4) / 2}\left(n_{1,4}-n_{-1,4}\right)+p^{m} \\
= & p^{m / 2}\left(n_{1,0}-n_{-1,0}\right)+p^{(m+2) / 2}\left(n_{1,2}-n_{-1,2}\right) \\
& +p^{(m+4) / 2}\left(n_{1,4}-n_{-1,4}\right)+p^{m} \\
= & p^{3 m}, \\
\sum & S(\alpha, \beta, \gamma)^{2} \\
\alpha, \beta, \gamma \in \mathbb{F}_{q} & p^{m}\left(n_{1,0}+n_{-1,0}\right)-p^{m+1}\left(n_{1,1}+n_{-1,1}\right) \\
& +p^{m+2}\left(n_{1,2}+n_{-1,2}\right)-p^{m+3}\left(n_{1,3}+n_{-1,3}\right) \\
& +p^{m+4}\left(n_{1,4}+n_{-1,4}\right)+p^{2 m} \\
= & p^{3 m}, \\
\sum & S(\alpha, \beta, \gamma)^{3} \\
\alpha, \beta, \gamma \in \mathbb{F}_{q} & +p^{3(m+4) / 2}\left(n_{1,4}-n_{-1,4}\right)+p^{3 m} \\
= & p^{3 m / 2}\left(n_{1,0}-n_{-1,0}\right)-i p^{3(m+1) / 2}\left(n_{1,1}-n_{-1,1}\right) \\
& +p^{3(m+2) / 2}\left(n_{1,2}-n_{-1,2}\right)-i p^{3(m+3) / 2}\left(n_{1,3}-n_{-1,3}\right) \\
& +p^{3(m+4) / 2}\left(n_{1,4}-n_{-1,4}\right)+p^{3 m} \\
= & p^{3 m / 2}\left(n_{1,0}-n_{-1,0}\right)+p^{3(m+2) / 2}\left(n_{1,2}-n_{-1,2}\right) \\
& \left(p^{3 m}\left(p^{m}-1\right)+1\right) p^{3 m}, \\
& S(13)
\end{aligned}
$$

where the first one comes from the fact that there are $p^{3 m}-1$ elements in the set $\mathbb{F}_{q}^{3} \backslash\{(0,0,0)\}$. Also, note that $S(\alpha, \beta, \gamma)=$ $p^{m}$ when $\alpha=\beta=\gamma=0$.

Using $n_{j}=n_{\varepsilon, j}=\left|N_{\varepsilon, j}\right|$ for $j=1,3$, the result is obtained by simplification.

3.2. The Fourth Moment of $S(\alpha, \beta, \gamma)$. For the fourth moment of $S(\alpha, \beta, \gamma)$ in the particular case of $p=3$, there is the following result about the number of solutions of the equation system:

$$
\begin{gathered}
x^{2}+y^{2}+z^{2}+1=0, \\
x^{p+1}+y^{p+1}+z^{p+1}+1=0, \\
x^{p^{2}+1}+y^{p^{2}+1}+z^{p^{2}+1}+1=0
\end{gathered}
$$

in Lemma 4 , which is denoted by $T_{4}$. 
Lemma 4 (see [19]). Let $p=3$ and $q=p^{m}$. Then

$$
T_{4}=4\left(2 p^{m}-3\right) \text {. }
$$

Using Lemma 4 and $M_{3}$ in Lemma $2, M_{4}$ is calculated in Lemma 5, where $M_{4}=M_{3}+(q-1) T_{4}$.

Lemma 5. Let $p=3$ and $q=p^{m}$. The number of solutions of the following equation system

$$
\begin{gathered}
x^{2}+y^{2}+z^{2}+w^{2}=0, \\
x^{p+1}+y^{p+1}+z^{p+1}+w^{p+1}=0, \\
x^{p^{2}+1}+y^{p^{2}+1}+z^{p^{2}+1}+w^{p^{2}+1}=0
\end{gathered}
$$$$
\text { is } M_{4}=8\left(p^{m}-1\right)^{2}+1 \text {. }
$$

Corresponding to Lemma 2, the result of the fourth moment is provided in Lemma 6 by applying Lemma 5 .

Lemma 6. Let $p=3$ and $q=p^{m}$. Then

$$
\sum_{\alpha, \beta, \gamma \in \mathbb{F}_{q}} S(\alpha, \beta, \gamma)^{4}=M_{4} \cdot p^{3 m}=\left(8\left(p^{m}-1\right)^{2}+1\right) p^{3 m} .
$$

Corresponding to Lemma 3, Lemma 6 can be rewritten as the following corollary using the symbols of (10) and (11).

Corollary 7. Let $p=3$ and $q=p^{m}$, where $m$ is an even integer. Then

$$
\begin{aligned}
& n_{1,0}+n_{-1,0}+2 n_{1} p^{2}+p^{4}\left(n_{1,2}+n_{-1,2}\right)+2 n_{3} p^{6} \\
& \quad+p^{8}\left(n_{1,4}+n_{-1,4}\right)=\left(8\left(p^{m}-1\right)^{2}-p^{m}+1\right) p^{m} .
\end{aligned}
$$

3.3. The Fifth Moment of $S(\alpha, \beta, \gamma)$. For the fifth moment of $S(\alpha, \beta, \gamma)$, we need Magma [28] to find the number of solutions of the following equation system:

$$
\begin{gathered}
x^{2}+y^{2}+z^{2}+w^{2}+u^{2}=0, \\
x^{p+1}+y^{p+1}+z^{p+1}+w^{p+1}+u^{p+1}=0, \\
x^{p^{2}+1}+y^{p^{2}+1}+z^{p^{2}+1}+w^{p^{2}+1}+u^{p^{2}+1}=0,
\end{gathered}
$$

which is denoted by $M_{5}$.

The irreducible components corresponding to the projective variety defined by (19) are listed in Table 1 using Magma [28]. Every block of Table 1 contains a system of three equations (" $=0$ " is omitted), the solutions of which satisfy (19). The union of all the solutions in each block presents the solutions of (19) exactly. Those equation systems are circulant symmetric about the variables. In general, few works are provided to deal with the moments using five variables. In this paper, Magma helps us on the reduction of such systems in Lemmas 8 and 9 and Corollary 10. For relevant knowledge of algebraic geometry, the reader is referred to [29].

Lemma 8. Let $p=3$ and $q=p^{m}$. Then

$$
M_{5}=5\left(p^{m}-1\right)\left(8 p^{m}-2 p-10\right)+1 \text {. }
$$

Proof. The number of nonzero solutions of $x^{2}+y^{2}=x^{p+1}+$ $y^{p+1}=x^{p^{2}+1}+y^{p^{2}+1}=0$ is $M_{2}^{\prime}=M_{2}-1=0$, where $M_{2}=1$ [19], and the number of nonzero elements in

$$
\begin{aligned}
& M_{3}=\#\left\{(x, y, z) \in \mathbb{F}_{q}^{3} \mid x^{2}+y^{2}+z^{2}=0,\right. \\
& x^{p+1}+y^{p+1}+z^{p+1}=0, \\
&\left.x^{p^{2}+1}+y^{p^{2}+1}+z^{p^{2}+1}=0\right\} \\
&=(p+1)(q-1)+1
\end{aligned}
$$

is $M_{3}^{\prime}=(p+1)(q-1)$. From Lemma 5, the number of nonzero solutions of (16) is $M_{4}^{\prime}=M_{4}-4 M_{3}^{\prime}-1=\left(p^{m}-1\right)\left(8 p^{m}-\right.$ $4 p-12)$

For the solutions of equation system (19), Table 1 shows that at least one of the elements $x, y, z, w, u$ is zero, and there are two cases to be considered.

(i) If only one of the five variables is zero, the number of such solutions is $5 M_{4}^{\prime}=5\left(p^{m}-1\right)\left(8 p^{m}-4 p-12\right)$.

(ii) If two variables are zero, the number of such solutions is $\left(\begin{array}{l}5 \\ 2\end{array}\right) M_{3}^{\prime}=10(p+1)(q-1)$.

Altogether, the number of solutions of equation system (19) is

$$
5 M_{4}^{\prime}+10 M_{3}^{\prime}+1=5\left(p^{m}-1\right)\left(8 p^{m}-2 p-10\right)+1 .
$$

Applying Lemma 8, the result about the fifth moment of exponential sum $S(\alpha, \beta, \gamma)$ is obtained.

Lemma 9. Let $p=3$ and $q=p^{m}$. Then

$$
\begin{aligned}
\sum_{\alpha, \beta, \gamma \in \mathbb{F}_{q}} S(\alpha, \beta, \gamma)^{5} & =M_{5} \cdot p^{3 m} \\
& =\left(5\left(p^{m}-1\right)\left(8 p^{m}-2 p-10\right)+1\right) p^{3 m} .
\end{aligned}
$$

Corollary 10. Let $p=3$ and $q=p^{m}$, where $m$ is an even integer. Then

$$
\begin{aligned}
n_{1,0} & -n_{-1,0}+p^{5}\left(n_{1,2}-n_{-1,2}\right)+p^{10}\left(n_{1,4}-n_{-1,4}\right) \\
& =\left(5\left(p^{m}-1\right)\left(8 p^{m}-2 p-10\right)-p^{2 m}+1\right) p^{m / 2} .
\end{aligned}
$$

3.4. MacWilliams' Identities. MacWilliams' theorem is for the Hamming weight enumerators of linear codes over finite field [30] (we consider prime field $\mathbb{F}_{p}$ ). Using this theorem, Lemma 12 is provided for the weight distribution using dual code's first few weights of Lemma 11. The two identities in Lemma 12 will combine with previous identities in final result.

Let $A_{i}$ be the number of codewords of weight $i$ in a code $C$ with length $l$ and dimension $k$, where $0 \leq i \leq l$. Let $A_{i}^{\prime}$ be the corresponding number in the dual code $C^{\perp}$. Then

$$
W_{C}(x, y)=\frac{1}{\left|C^{\perp}\right|} W_{C^{\perp}}(x+(p-1) y, x-y),
$$


TABLE 1: Irreducible components corresponding to the projective variety defined by (19).

\begin{tabular}{|c|c|c|c|c|c|c|c|}
\hline$\overline{x^{2}}$ & $x^{2}$ & $x^{2}$ & $x^{2}$ & $x^{2}$ & $x^{2}$ & $x^{2}$ & $x^{2}$ \\
\hline $\begin{array}{l}y-w-u \\
z-w+u\end{array}$ & $\begin{array}{l}y-w-u \\
z+w-u\end{array}$ & $\begin{array}{l}y-w+u \\
z-w-u\end{array}$ & $\begin{array}{l}y-w+u \\
z+w+u\end{array}$ & $\begin{array}{l}y+w-u \\
z-w-u\end{array}$ & $\begin{array}{l}y+w-u \\
z+w+u\end{array}$ & $\begin{array}{l}y+w+u \\
z-w+u\end{array}$ & $\begin{array}{l}y+w+u \\
z+w-u\end{array}$ \\
\hline$y^{2}$ & $y^{2}$ & $y^{2}$ & $y^{2}$ & $y^{2}$ & $y^{2}$ & $y^{2}$ & $y^{2}$ \\
\hline$x-w-u$ & $x-w-u$ & $x-w+u$ & $x-w+u$ & $x+w-u$ & $x+w-u$ & $x+w+u$ & $x+w+u$ \\
\hline$z-w+u$ & $z+w-u$ & $z-w-u$ & $z+w+u$ & $z-w-u$ & $z+w+u$ & $z-w+u$ & $z+w-u$ \\
\hline$\overline{z^{2}}$ & $z^{2}$, & $z^{2}$, & $z^{2}$ & $z^{2}$, & $z^{2}$ & $z^{2}$ & $z^{2}$ \\
\hline$x-w-u$ & $x-w-u$ & $x-w+u$ & $x-w+u$ & $x+w-u$ & $x+w-u$ & $x+w+u$ & $x+w+u$ \\
\hline$y-w+u$ & $y+w-u$ & $y-w-u$ & $y+w+u$ & $y-w-u$ & $y+w+u$ & $y-w+u$ & $y+w-u$ \\
\hline$w^{2}$ & $w^{2}$ & $w^{2}$ & $w^{2}$ & $w^{2}$ & $w^{2}$ & $w^{2}$ & $w^{2}$ \\
\hline$x-z-u$ & $x-z-u$ & $x-z+u$ & $x-z+u$ & $x+z-u$ & $x+z-u$ & $x+z+u$ & $x+z+u$ \\
\hline$y-z+u$ & $y+z-u$ & $y-z-u$ & $y+z+u$ & $y-z-u$ & $y+z+u$ & $y-z+u$ & $y+z-u$ \\
\hline$u^{2}$ & $u^{2}$ & $u^{2}$ & $u^{2}$ & $u^{2}$ & $u^{2}$ & $u^{2}$ & $u^{2}$ \\
\hline$x-z-w$ & $x-z-w$ & $x-z+w$ & $x-z+w$ & $x+z-w$ & $x+z-w$ & $x+z+w$ & $x+z+w$ \\
\hline$y-z+w$ & $y+z-w$ & $y-z-w$ & $y+z+w$ & $y-z-w$ & $y+z+w$ & $y-z+w$ & $y+z-w$ \\
\hline
\end{tabular}

where $W_{C}(x, y)=\sum_{i=0}^{l} A_{i} x^{l-i} y^{i}$. Setting $x=1$, (25) changes to

$$
\sum_{i=0}^{l} A_{i} y^{i}=\frac{1}{p^{l-k}} \sum_{i=0}^{l} A_{i}^{\prime}(1+(p-1) y)^{l-i}(1-y)^{i}
$$

Differentiating (26) with respect to $y$,

$$
\begin{aligned}
& \sum_{i=1}^{l} i A_{i} y^{i-1} \\
& \quad=\frac{1}{p^{l-k}} \sum_{i=0}^{l} A_{i}^{\prime}\left((l-i)(p-1)(1+(p-1) y)^{l-i-1}(1-y)^{i}\right. \\
& \left.\quad+(1+(p-1) y)^{l-i} i(-1)(1-y)^{i-1}\right) .
\end{aligned}
$$

Setting $y=1$, the first MacWilliams' moment identity is obtained for $l \geq 2$

$$
\sum_{i=1}^{l} \frac{i A_{i}}{p^{k}}=\frac{1}{p}\left((p-1) l-A_{1}^{\prime}\right)=\frac{1}{p}(p-1) l \quad \text { if } A_{1}^{\prime}=0 \text {. }
$$

Differentiating again,

$$
\begin{aligned}
& \sum_{i=1}^{l} i(i-1) A_{i} y^{i-2} \\
& =\frac{1}{p^{l-k}} \sum_{i=0}^{l} A_{i}^{\prime}\left\{(l-i)(l-i-1)(p-1)^{2}\right. \\
& \times(1+(p-1) y)^{l-i-2}(1-y)^{i}
\end{aligned}
$$

$$
\begin{aligned}
& +2(l-i)(p-1)(1+(p-1) y)^{l-i-1} \\
& \times i(-1)(1-y)^{i-1} \\
& \left.+(1+(p-1) y)^{l-i} i(i-1)(1-y)^{i-2}\right\} .
\end{aligned}
$$

Substituting $y=1$, the second MacWilliams' moment identity is obtained:

$$
\begin{array}{r}
\sum_{i=1}^{l} i(i-1) A_{i}=\frac{1}{p^{l-k}}\left(l(l-1)(p-1)^{2} p^{l-2}+\right. \\
\left.2 A_{2}^{\prime} p^{l-2}\right) \\
\text { if } A_{1}^{\prime}=0 .
\end{array}
$$

Differentiating for the third and fourth time, if $A_{1}^{\prime}=A_{3}^{\prime}=0$, the fourth MacWilliams' moment identity is

$$
\begin{aligned}
& \sum_{i=1}^{l} i(i-1)(i-2)(i-3) A_{i} \\
& \quad=\frac{1}{p^{l-k}}\left(l(l-1)(l-2)(l-3) p^{l-4}(p-1)^{4}\right. \\
& \left.\quad+12 A_{2}^{\prime}(l-2)(l-3)(p-1)^{2} p^{l-4}+24 A_{4}^{\prime} p^{l-4}\right) .
\end{aligned}
$$

Lemma 11. Let $p=3, q=p^{m}$. Let $\mathscr{C}$ denote the cyclic code with nonzeros $\pi^{-2}, \pi^{-(p+1)}$, and $\pi^{-\left(p^{2}+1\right)}$; the weights of the dual code $\mathscr{C}^{\perp}$ satisfy the following:

$$
\begin{aligned}
& A_{0}^{\prime}=1, \quad A_{1}^{\prime}=0, \quad A_{2}^{\prime}=p^{m}-1, \quad A_{3}^{\prime}=0, \\
& A_{4}^{\prime}=\frac{\left(p^{m}-1\right)\left(2 p^{m}-p-3\right)}{3}+\frac{\left(p^{m}-1\right)\left(p^{m}-3\right)}{2} .
\end{aligned}
$$


TABLE 2: Irreducible components corresponding to (37).

\begin{tabular}{lllll}
\hline$y^{4}$, & $y^{4}$, & $z^{4}$, & $z^{4}$, & $z^{4}$, \\
$x^{2}+y^{2}$, & $x^{2}+y^{2}$, & $x^{2}+z^{2}$, & $x^{2}+z^{2}$, & $y^{2}+z^{2}$, \\
$z-w$, & $z+w$, & $y-w$, & $y+w$, & $x-w$, \\
\hline$z^{4}$, & $w^{4}$, & $w^{4}$, & $w^{4}$ & $w^{4}$, \\
$y^{2}+z^{2}$, & $y^{2}-y z+z^{2}+w^{2}$, & $y^{2}-y z+z^{2}+w^{2}$, & $y^{2}+y z+z^{2}+w^{2}$, & $y^{2}+y z+z^{2}+w^{2}$, \\
$x+w$, & $x-y+z$, & $x+y-z$, & $x-y-z$, & $x+y+z$, \\
\hline
\end{tabular}

Proof. Below, codewords are considered in the dual code. It is easy to see that $A_{0}^{\prime}=1$ and $A_{1}^{\prime}=0$. For the codewords with weight two, if the components at the two positions have the same value, we find that $M_{2}^{\prime}=0$. Let us consider the following equation system about the positions:

$$
\begin{gathered}
x^{2}-y^{2}=0, \\
x^{p+1}-y^{p+1}=0, \\
x^{p^{2}+1}-y^{p^{2}+1}=0,
\end{gathered}
$$

which should be satisfied by the coordinates of the codewords. For any $y \in \mathbb{F}_{q}^{*}, x=-y$ is the other corresponding coordinate. That is, $A_{2}^{\prime}=p^{m}-1$.

As to weight-three codewords, there are two cases to be considered.

(i) If all the values corresponding to the three coordinates of the codewords are the same, then study the solutions of the following equation system:

$$
\begin{gathered}
x^{2}+y^{2}+1=0, \\
x^{p+1}+y^{p+1}+1=0, \\
x^{p^{2}+1}+y^{p^{2}+1}+1=0 .
\end{gathered}
$$

From the first two equations of (34), we find that $x^{2}=$ $y^{2}=1$ contradicting the fact that $x, y, 1$ should be different.

(ii) If one value is different from the other two, consider

$$
\begin{gathered}
x^{2}-y^{2}+1=0, \\
x^{p+1}-y^{p+1}+1=0, \\
x^{p^{2}+1}-y^{p^{2}+1}+1=0 .
\end{gathered}
$$

Solving the above system, we have $x=0$ contradicting the fact that the coordinates should be different from 0 .

Combing the above two cases, $A_{3}^{\prime}=0$.

Now, let us consider the number of codewords with weight four in three cases.

Case I. At the four positions, the components have the same value. According to the proof of Lemma 8, the number of nonzero solutions of equation system $(16)$ is $M_{4}^{\prime}=\left(p^{m}-1\right)$ $\left(8 p^{m}-4 p-12\right)$. For a solution $(x, y, z, w)$ of $(16)$, if two of them are equal, for example, $z=w=v$, then (16) becomes

$$
\begin{gathered}
x^{2}+y^{2}-v^{2}=0, \\
x^{p+1}+y^{p+1}-v^{p+1}=0, \\
x^{p^{2}+1}+y^{p^{2}+1}-v^{p^{2}+1}=0 .
\end{gathered}
$$

Solving the above system, it can be found that $x$ or $y$ is zero, so the number of nonzero solutions of (36) is 0 . Then all those $M_{4}^{\prime}$ nonzero solutions of (16) correspond to the codewords, where $24=4$ ! solutions correspond to a four-tuple and each tuple corresponds to two codewords. Therefore, there are 2 . $M_{4}^{\prime} / 24=M_{4}^{\prime} / 12$ codewords.

Case II. One value at the four nonzero positions is different from the other three values. Consider the solutions of the following system:

$$
\begin{gathered}
x^{2}+y^{2}+z^{2}-w^{2}=0, \\
x^{p+1}+y^{p+1}+z^{p+1}-w^{p+1}=0, \\
x^{p^{2}+1}+y^{p^{2}+1}+z^{p^{2}+1}-w^{p^{2}+1}=0 .
\end{gathered}
$$

Using Mamga [28], the irreducible components of the projective variety defined by (37) are provided by the polynomials listed in Table 2. It is easy to see that at least one of $x, y, z, w$ is zero, so the solutions can not correspond to codewords.

Case III. Two values at the coordinates are the same. Let us consider

$$
\begin{gathered}
x^{2}+y^{2}-z^{2}-w^{2}=0, \\
x^{p+1}+y^{p+1}-z^{p+1}-w^{p+1}=0, \\
x^{p^{2}+1}+y^{p^{2}+1}-z^{p^{2}+1}-w^{p^{2}+1}=0 .
\end{gathered}
$$

Again the irreducible components are presented in Table 3, by which only the cases $x=-z, y=-w$, and $x=-w$, $y=-z$ are the possible solutions which can correspond to codewords since coordinates should be different. The number of such solutions is $2\left(p^{m}-1\right)\left(p^{m}-3\right)$ which corresponds to $4 \cdot\left(2\left(p^{m}-1\right)\left(p^{m}-3\right) / 16\right)=\left(p^{m}-1\right)\left(p^{m}-3\right) / 2$ codewords, since every four-tuple $\left(x_{0}, y_{0}, z_{0}, w_{0}\right)$ corresponds to $4 \cdot 2 \cdot 2$ solutions of (38). In fact, if $c$ is a weight-four codeword with nonzero positions and values $\left(x_{0}, y_{0},-x_{0},-y_{0}\right) \rightarrow(1,1,-1,-1)$, then $\left(x_{0}, y_{0},-x_{0},-y_{0}\right) \rightarrow(-1,-1,1,1),\left(x_{0}, y_{0},-x_{0},-y_{0}\right) \rightarrow$ $(-1,1,1,-1)$, and $\left(x_{0}, y_{0},-x_{0},-y_{0}\right) \rightarrow(1,-1,-1,1)$ can all represent weight-four codewords. 
TABLE 3: Irreducible components corresponding to (38).

\begin{tabular}{|c|c|c|c|c|c|c|c|}
\hline $\begin{array}{l}x-z \\
y-w\end{array}$ & $\begin{array}{l}x-z \\
y+w\end{array}$ & $\begin{array}{l}x+z \\
y-w\end{array}$ & $\begin{array}{l}x+z \\
y+w\end{array}$ & $\begin{array}{l}x-w \\
y-z\end{array}$ & $\begin{array}{l}x-w \\
y+z\end{array}$ & $\begin{array}{c}x+w \\
y-z\end{array}$ & $\begin{array}{l}x+w \\
y+z\end{array}$ \\
\hline
\end{tabular}

Combing the above three cases,

$$
\begin{aligned}
A_{4}^{\prime} & =\frac{M_{4}^{\prime}}{12}+\frac{\left(p^{m}-1\right)\left(p^{m}-3\right)}{2} \\
& =\frac{\left(p^{m}-1\right)\left(2 p^{m}-p-3\right)}{3}+\frac{\left(p^{m}-1\right)\left(p^{m}-3\right)}{2} .
\end{aligned}
$$

The result of the lemma is obtained.

Lemma 12. Let $p=3, q=p^{m}$, where $m \geq 6$ is an even integer. The notations defined in (10) and (11) satisfy

$$
\begin{aligned}
& n_{1,0}+n_{-1,0}+p^{2}\left(n_{1,2}+n_{-1,2}\right)+p^{4}\left(n_{1,4}+n_{-1,4}\right)=a, \\
& n_{1,0}+n_{-1,0}+p^{4}\left(n_{1,2}+n_{-1,2}\right)+p^{8}\left(n_{1,4}+n_{-1,4}\right)=b,
\end{aligned}
$$

where

$$
\begin{aligned}
& a=\left(p^{3 m-2}\left(\left(p^{m}-1\right)\left(p^{m}-2\right)(p-1)^{2}+2 A_{2}^{\prime}\right)\right. \\
& -p^{2(m-1)}(p-1)^{2}\left(p^{3 m}-2 p^{2 m}+1\right) \\
& \left.+(p-1)\left(p^{m}-1\right) p^{3 m-1}\right) \times\left((p-1)^{2} p^{m-2}\right)^{-1}, \\
& b=\left\{p ^ { 3 m - 4 } \left(\left(p^{m}-1\right)\left(p^{m}-2\right)\left(p^{m}-3\right)\left(p^{m}-4\right)(p-1)^{4}\right.\right. \\
& \left.+12 A_{2}^{\prime}\left(p^{m}-3\right)\left(p^{m}-4\right)(p-1)^{2}+24 A_{4}^{\prime}\right) \\
& -(p-1)^{5} p^{3(m-1)} a-p^{4(m-1)}(p-1)^{4} \\
& \quad+\left(p^{3 m}-4 p^{2 m}-16 p^{m}+19\right) \\
& +6\left((p-1)^{3} p^{2(m-1)} a+p^{3(m-1)}(p-1)^{3}\right. \\
& \left.\quad \times\left(p^{3 m}-p^{2 m+1}-4 p^{m}+6\right)\right) \\
& \left.+6\left((p-1)\left(p^{m}-1\right) p^{3 m-1}\right)\right\} \times\left((p-1)^{4} p^{2 m-4}\right)^{-1}, \\
& +11\left((p-1)^{2} p^{m-2} a\right. \\
& \left.+p^{2(m-1)}(p-1)^{2}\left(p^{3 m}-2 p^{2 m}+1\right)\right) \\
& +6
\end{aligned}
$$

and $A_{2}^{\prime}, A_{4}^{\prime}$ are defined in Lemma 11.

Proof. Define the following notations for simplification:

$$
\begin{array}{cc}
R_{00}=p^{m-1}(p-1), & R_{0}=\frac{p-1}{p} p^{m / 2}, \\
R_{2}=\frac{p-1}{p} p^{(m+2) / 2}, & R_{4}=\frac{p-1}{p} p^{(m+4) / 2} .
\end{array}
$$

The use of MacWilliams' identities in the following paragraphs implies the condition $m \geq 6$; refer to [26, Lemma 10]. By (4) and [26, Lemma 9], $\mathscr{C}$ has seven possible nonzero weights:

$$
\begin{array}{ccc}
A_{R_{00}}=2\left(n_{1}+n_{3}\right), & A_{R_{00}-R_{0}}=n_{1,0}, & A_{R_{00}+R_{0}}=n_{-1,0}, \\
A_{R_{00}-R_{2}}=n_{1,2}, & A_{R_{00}+R_{2}}=n_{-1,2}, & A_{R_{00}-R_{4}}=n_{1,4}, \\
& A_{R_{00}+R_{4}}=n_{-1,4} . &
\end{array}
$$

With the above notations, the first four moments of codeword weights can be computed:

$$
\begin{aligned}
\sum_{i=0}^{l} i A_{i} & \\
= & R_{00} 2\left(n_{1}+n_{3}\right)+\left(R_{00}-R_{0}\right) n_{1,0}+\left(R_{00}+R_{0}\right) n_{-1,0} \\
& +\left(R_{00}-R_{2}\right) n_{1,2}+\left(R_{00}+R_{2}\right) n_{-1,2}+\left(R_{00}-R_{4}\right) n_{1,4} \\
& +\left(R_{00}+R_{4}\right) n_{-1,4} \\
= & R_{00}\left(2\left(n_{1}+n_{3}\right)+n_{1,0}+n_{-1,0}+n_{1,2}+n_{-1,2}+n_{1,4}+n_{-1,4}\right) \\
& \quad-\left(R_{0}\left(n_{1,0}-n_{-1,0}\right)+R_{2}\left(n_{1,2}-n_{-1,2}\right)+R_{4}\left(n_{1,4}-n_{-1,4}\right)\right) \\
= & \left(p^{3 m}-1\right) p^{m-1}(p-1)-\frac{p-1}{p} p^{m / 2}\left(p^{m / 2}\left(p^{2 m}-1\right)\right) \\
= & (p-1)\left(p^{m}-1\right) p^{3 m-1}, \\
& \quad+R_{0}^{2}\left(n_{1,0}+n_{-1,0}\right)+R_{2}^{2}\left(n_{1,2}+n_{-1,2}\right)+R_{4}^{2}\left(n_{1,4}+n_{-1,4}\right) \\
& \quad+R_{00}^{2}\left(2\left(n_{1}+n_{3}\right)+n_{1,0}+n_{-1,0}+n_{1,2}+n_{-1,2}+n_{1,4}+n_{-1,4}\right) \\
& \quad+\left(R_{00}-R_{2}\right)^{2} n_{1,2}+\left(R_{00}+R_{2}\right)^{2} n_{-1,2}+\left(n_{1,0}-n_{-1,0}\right)+R_{2}\left(n_{1,2}-n_{-1,2}\right) \\
\sum_{i=0}^{l} i^{2} A_{i} & R_{00}^{2} 2\left(n_{1}+n_{1,4}\right)+\left(R_{00}-R_{0}\right)^{2} n_{1,0}+\left(R_{00}+R_{0}\right)^{2} n_{-1,0}
\end{aligned}
$$




$$
\begin{aligned}
& =(p-1)^{2} p^{m-2} \\
& \times\left(n_{1,0}+n_{-1,0}+p^{2}\left(n_{1,2}+n_{-1,2}\right)+p^{4}\left(n_{1,4}+n_{-1,4}\right)\right) \\
& +p^{2(m-1)}(p-1)^{2}\left(p^{3 m}-2 p^{2 m}+1\right) \\
& \sum_{i=0}^{l} i^{3} A_{i} \\
& =R_{00}^{3} 2\left(n_{1}+n_{3}\right)+\left(R_{00}-R_{0}\right)^{3} n_{1,0}+\left(R_{00}+R_{0}\right)^{3} n_{-1,0} \\
& +\left(R_{00}-R_{2}\right)^{3} n_{1,2}+\left(R_{00}+R_{2}\right)^{3} n_{-1,2}+\left(R_{00}-R_{4}\right)^{3} n_{1,4} \\
& +\left(R_{00}+R_{4}\right)^{3} n_{-1,4} \\
& =R_{00}^{3}\left(2\left(n_{1}+n_{3}\right)+n_{1,0}+n_{-1,0}+n_{1,2}+n_{-1,2}+n_{1,4}+n_{-1,4}\right) \\
& -3 R_{00}^{2}\left(R_{0}\left(n_{1,0}-n_{-1,0}\right)+R_{2}\left(n_{1,2}-n_{-1,2}\right)\right. \\
& \left.+R_{4}\left(n_{1,4}-n_{-1,4}\right)\right) \\
& +3 R_{00}\left(R_{0}^{2}\left(n_{1,0}+n_{-1,0}\right)+R_{2}^{2}\left(n_{1,2}+n_{-1,2}\right)\right. \\
& \left.+R_{4}^{2}\left(n_{1,4}+n_{-1,4}\right)\right) \\
& -\left(R_{0}^{3}\left(n_{1,0}-n_{-1,0}\right)+R_{2}^{3}\left(n_{1,2}-n_{-1,2}\right)+R_{4}^{3}\left(n_{1,4}-n_{-1,4}\right)\right) \\
& =(p-1)^{3} p^{2(m-1)} \\
& \times\left(n_{1,0}+n_{-1,0}+p^{2}\left(n_{1,2}+n_{-1,2}\right)+p^{4}\left(n_{1,4}+n_{-1,4}\right)\right) \\
& +p^{3(m-1)}(p-1)^{3}\left(p^{3 m}-p^{2 m+1}-4 p^{m}+6\right),
\end{aligned}
$$

$$
\begin{aligned}
& \times\left(n_{1,0}+n_{-1,0}+p^{4}\left(n_{1,2}+n_{-1,2}\right)+p^{8}\left(n_{1,4}+n_{-1,4}\right)\right) \\
& +p^{4(m-1)}(p-1)^{4}\left(p^{3 m}-4 p^{2 m}-16 p^{m}+19\right) .
\end{aligned}
$$

From (30), (44), and (45) we have

$$
\begin{aligned}
\sum_{i=1}^{l} i(i-1) A_{i}= & \frac{1}{p^{l-k}}\left(l(l-1)(p-1)^{2} p^{l-2}+2 A_{2}^{\prime} p^{l-2}\right) \\
= & p^{3 m-2}\left(l(l-1)(p-1)^{2}+2 A_{2}^{\prime}\right) \\
= & \sum_{i=1}^{l} i^{2} A_{i}-\sum_{i=1}^{l} i A_{i} \\
= & (p-1)^{2} p^{m-2}\left(n_{1,0}+n_{-1,0}+p^{2}\left(n_{1,2}+n_{-1,2}\right)\right. \\
& +p^{2(m-1)}(p-1)^{2}\left(p^{3 m}-2 p^{2 m}+1\right) \\
& -(p-1)\left(p^{m}-1\right) p^{3 m-1},
\end{aligned}
$$

and the first one of (40) is obtained after simplification.

According to the MacWilliams' fourth moment identity (31), (44), (45), (46), and (47),

$$
\begin{aligned}
& \sum_{i=0}^{l} i^{4} A_{i} \\
& =R_{00}^{4} 2\left(n_{1}+n_{3}\right)+\left(R_{00}-R_{0}\right)^{4} n_{1,0}+\left(R_{00}+R_{0}\right)^{4} n_{-1,0} \\
& \quad+\left(R_{00}-R_{2}\right)^{4} n_{1,2}+\left(R_{00}+R_{2}\right)^{4} n_{-1,2}+\left(R_{00}-R_{4}\right)^{4} n_{1,4} \\
& \quad+\left(R_{00}+R_{4}\right)^{4} n_{-1,4} \\
& =R_{00}^{4}\left(2\left(n_{1}+n_{3}\right)+n_{1,0}+n_{-1,0}+n_{1,2}+n_{-1,2}+n_{1,4}+n_{-1,4}\right) \\
& \quad-4 R_{00}^{3}\left(R_{0}\left(n_{1,0}-n_{-1,0}\right)+R_{2}\left(n_{1,2}-n_{-1,2}\right)\right. \\
& \left.\quad+R_{4}\left(n_{1,4}-n_{-1,4}\right)\right) \\
& \quad+6 R_{00}^{2}\left(R_{0}^{2}\left(n_{1,0}+n_{-1,0}\right)+R_{2}^{2}\left(n_{1,2}+n_{-1,2}\right)\right. \\
& \left.\quad+R_{4}^{2}\left(n_{1,4}+n_{-1,4}\right)\right) \\
& \quad+4 R_{00}\left(R_{0}^{3}\left(n_{1,0}-n_{-1,0}\right)+R_{2}^{3}\left(n_{1,2}-n_{-1,2}\right)\right. \\
& \left.\quad+R_{4}^{3}\left(n_{1,4}-n_{-1,4}\right)\right) \\
& \quad\left(R_{0}^{4}\left(n_{1,0}+n_{-1,0}\right)+R_{2}^{4}\left(n_{1,2}+n_{-1,2}\right)+R_{4}^{4}\left(n_{1,4}+n_{-1,4}\right)\right)
\end{aligned}
$$




$$
\begin{aligned}
& -6\left((p-1)^{3} p^{2(m-1)} a\right. \\
& \left.\quad+p^{3(m-1)}(p-1)^{3}\left(p^{3 m}-p^{2 m+1}-4 p^{m}+6\right)\right) \\
& +11\left((p-1)^{2} p^{m-2} a+p^{2(m-1)}(p-1)^{2}\left(p^{3 m}-2 p^{2 m}+1\right)\right) \\
& -6\left((p-1)\left(p^{m}-1\right) p^{3 m-1}\right)
\end{aligned}
$$

3.5. Weight Distribution of $\mathscr{C}$. In this subsection, the parameters defined in (10) and (11) are calculated in Lemma 13, and the weight distribution of the cyclic code $\mathscr{C}$ is determined in Theorem 15.

Lemma 13. Let $p=3, q=p^{m}$ where $m \geq 6$ is an even integer. The notations defined in (10) and (11) satisfy

$$
\begin{aligned}
& n_{1,0}=\left(-\left(b+c_{6}-a p^{2}-a p^{3}-a p^{4}-a p^{5}-b p^{2}+c_{1} p^{6}\right.\right. \\
& \left.\left.+c_{2} p^{6}+c_{3} p^{3}+c_{3} p^{5}-c_{4} p^{2}-c_{4} p^{4}+c_{5} p^{2}\right)\right) \\
& \times\left(-2 p^{6}+2 p^{4}+2 p^{2}-2\right)^{-1} \\
& n_{-1,0}=\left(-\left(b-c_{6}-a p^{2}-a p^{3}-a p^{4}-a p^{5}-b p^{2}+c_{1} p^{6}\right.\right. \\
& \left.\left.-c_{2} p^{6}+c_{3} p^{3}+c_{3} p^{5}+c_{4} p^{2}+c_{4} p^{4}+c_{5} p^{2}\right)\right) \\
& \times\left(-2 p^{6}+2 p^{4}+2 p^{2}-2\right)^{-1} \\
& 2 n_{1}=\frac{-\left(b-c_{5}+a p^{3}-c_{3} p^{3}\right)}{\left(p^{2}-p^{4}\right)}, \\
& n_{1,2}=\left(c_{4}-c_{6}+a p-c_{5} p+a p^{2}+a p^{4}+a p^{5}-c_{1} p^{5}\right. \\
& \left.-c_{2} p^{4}-c_{3} p^{2}-c_{3} p^{4}+c_{4} p^{4}\right) \\
& \times\left(2 p^{7}-4 p^{5}+2 p^{3}\right)^{-1} \\
& n_{-1,2}=\left(-\left(c_{4}-c_{6}-a p+c_{5} p-a p^{2}-a p^{4}-a p^{5}+c_{1} p^{5}\right.\right. \\
& \left.\left.-c_{2} p^{4}+c_{3} p^{2}+c_{3} p^{4}+c_{4} p^{4}\right)\right) \\
& \times\left(2 p^{7}-4 p^{5}+2 p^{3}\right)^{-1}, \\
& 2 n_{3}=\frac{\left(b-c_{5}+a p-c_{3} p\right)}{\left(p^{4}-p^{6}\right)},
\end{aligned}
$$

$$
\begin{aligned}
n_{1,4}= & \left(-\left(b+c_{4}-c_{5}-c_{6}+a p-c_{3} p+a p^{2}+a p^{3}+a p^{4}\right.\right. \\
& \left.\left.-b p^{2}-c_{1} p^{4}-c_{2} p^{2}-c_{3} p^{3}+c_{4} p^{2}\right)\right) \\
& \times\left(2 p^{10}-2 p^{8}-2 p^{6}+2 p^{4}\right)^{-1}, \\
n_{-1,4}= & \left(b-c_{4}-c_{5}+c_{6}+a p-c_{3} p+a p^{2}+a p^{3}+a p^{4}\right. \\
& \left.\left.\quad-b p^{2}-c_{1} p^{4}+c_{2} p^{2}-c_{3} p^{3}-c_{4} p^{2}\right)\right) \\
& \times\left(2 p^{10}-2 p^{8}-2 p^{6}+2 p^{4}\right)^{-1},
\end{aligned}
$$

where

$$
\begin{gathered}
c_{1}=p^{3 m}-1, \quad c_{2}=p^{m / 2}\left(p^{2 m}-1\right), \\
c_{3}=p^{m}\left(p^{m}-1\right), \quad c_{4}=(p+1) p^{3 m / 2}\left(p^{m}-1\right), \\
c_{5}=\left(8\left(p^{m}-1\right)^{2}-p^{m}+1\right) p^{m}, \\
c_{6}=\left(5\left(p^{m}-1\right)\left(8 p^{m}-2 p-10\right)-p^{2 m}+1\right) p^{m / 2},
\end{gathered}
$$

and $a, b$ are defined in Lemma 12.

Proof. From Lemma 3, Corollaries 7 and 10, and Lemma 12, the following equations hold:

$$
\begin{gathered}
2\left(n_{1}+n_{3}\right)+n_{-1,0}+n_{1,0}+n_{-1,2}+n_{1,2}+n_{1,4}+n_{-1,4}=c_{1}, \\
n_{1,0}-n_{-1,0}+p\left(n_{1,2}-n_{-1,2}\right)+p^{2}\left(n_{1,4}-n_{-1,4}\right)=c_{2} \\
-2\left(p n_{1}+p^{3} n_{3}\right)+n_{1,0}+n_{-1,0}+p^{2}\left(n_{1,2}+n_{-1,2}\right) \\
\quad+p^{4}\left(n_{1,4}+n_{-1,4}\right)=c_{3}, \\
n_{1,0}-n_{-1,0}+p^{3}\left(n_{1,2}-n_{-1,2}\right)+p^{6}\left(n_{1,4}-n_{-1,4}\right)=c_{4}, \\
n_{1,0}+n_{-1,0}+2 n_{1} p^{2}+p^{4}\left(n_{1,2}+n_{-1,2}\right)+2 n_{3} p^{6} \\
\quad+p^{8}\left(n_{1,4}+n_{-1,4}\right)=c_{5}, \\
n_{1,0}-n_{-1,0}+p^{5}\left(n_{1,2}-n_{-1,2}\right)+p^{10}\left(n_{1,4}-n_{-1,4}\right)=c_{6}, \\
n_{1,0}+n_{-1,0}+p^{2}\left(n_{1,2}+n_{-1,2}\right)+p^{4}\left(n_{1,4}+n_{-1,4}\right)=a \\
n_{1,0}+n_{-1,0}+p^{4}\left(n_{1,2}+n_{-1,2}\right)+p^{8}\left(n_{1,4}+n_{-1,4}\right)=b .
\end{gathered}
$$

Solving the above equation system, the result is obtained.

Lemma 14. Let $p=3, q=p^{m}$, where $m \geq 6$ is an even integer. The number of solutions of the equation system

$$
\begin{gathered}
x^{2}+y^{2}+z^{2}+w^{2}+u^{2}+v^{2}=0, \\
x^{p+1}+y^{p+1}+z^{p+1}+w^{p+1}+u^{p+1}+v^{p+1}=0, \\
x^{p^{2}+1}+y^{p^{2}+1}+z^{p^{2}+1}+w^{p^{2}+1}+u^{p^{2}+1}+v^{p^{2}+1}=0,
\end{gathered}
$$


is

$$
\begin{gathered}
n_{1,0}+n_{-1,0}+p^{6}\left(n_{1,2}+n_{-1,2}\right)+p^{12}\left(n_{1,4}+n_{-1,4}\right) \\
-p^{3}\left(n_{1,1}+n_{-1,1}\right)-p^{9}\left(n_{1,3}+n_{-1,3}\right)+p^{3 m}
\end{gathered}
$$

Proof. The sixth moment of exponential sum $S(\alpha, \beta, \gamma)$ satisfies

$$
\begin{aligned}
\sum_{\alpha, \beta, \gamma \in \mathbb{F}_{q}} S(\alpha, \beta, \gamma)^{6} & \\
= & p^{3 m}\left(n_{1,0}+n_{-1,0}\right)+p^{3(m+2)}\left(n_{1,2}+n_{-1,2}\right) \\
& +p^{3(m+4)}\left(n_{1,4}+n_{-1,4}\right)-p^{3(m+1)}\left(n_{1,1}+n_{-1,1}\right) \\
& -p^{3(m+3)}\left(n_{1,3}+n_{-1,3}\right)+p^{6 m} \\
= & M_{6} \cdot p^{3 m}
\end{aligned}
$$

where $M_{6}$ is the number of solutions of (53). Solving the above equation for $M_{6}$, the result is obtained.

Equation (53) considers the case for 6 variables. Using the seventh moment of $S(\alpha, \beta, \gamma)$, the number of solutions can be calculated when there are 7 variables and so forth.

Theorem 15. Let $p=3, q=p^{m}$, where $m \geq 6$ is an even integer. The cyclic code $\mathscr{C}$ with nonzeros $\pi^{-2}, \pi^{-(p+1)}$ and $\pi^{-\left(p^{2}+1\right)}$ has seven nonzero weights:

$$
\begin{aligned}
A_{p^{m-1}(p-1)}= & 2\left(n_{1}+n_{3}\right), \quad A_{p^{m-1}(p-1)-((p-1) / p) p^{m / 2}}=n_{1,0} \\
& A_{p^{m-1}(p-1)+((p-1) / p) p^{m / 2}}=n_{-1,0} \\
& A_{p^{m-1}(p-1)-((p-1) / p) p^{(m+2) / 2}}=n_{1,2}, \\
& A_{p^{m-1}(p-1)+((p-1) / p) p^{(m+2) / 2}}=n_{-1,2} \\
& A_{p^{m-1}(p-1)-((p-1) / p) p^{(m+4) / 2}}=n_{1,4} \\
& A_{p^{m-1}(p-1)+((p-1) / p) p^{(m+4) / 2}}=n_{-1,4}
\end{aligned}
$$

where $\pi$ is a primitive element of the finite field $\mathbb{F}_{q}$.

It is interesting to consider the symmetric property about the weights of the cyclic code $\mathscr{C}$. If there is a weight of the form $p^{m-1}(p-1)+((p-1) / p) p^{(m+2 i) / 2}(i=0,1,2)$, then there is a weight of the form $p^{m-1}(p-1)-((p-1) / p) p^{(m+2 i) / 2}$. It seems that the weights are symmetric about the value $p^{m-1}(p-1)$ which is also a weight of $\mathscr{C}$. This phenomenon may be explained by the fact that, for symmetric matrices with corresponding quadratic residue $\Delta$, there are symmetric matrices with corresponding nonresidue $\Delta^{\prime}$ (Lemma 1). As the following example illustrates, in general the higher the value $i$ the less the number of corresponding weights.

Example 16. Let $p=3, q=p^{m}$, where $m=6$. The cyclic code $\mathscr{C}$ has nonzeros $\pi^{-2}, \pi^{-(p+1)}$, and $\pi^{-\left(p^{2}+1\right)}$, where $\pi$ is a primitive element of the finite field $\mathbb{F}_{q}$. Using Theorem 15, it has seven nonzero weights:

$$
\begin{gathered}
A_{486}=124245576, \quad A_{468}=128432304, \\
A_{504}=119277522, \quad A_{432}=8591310, \\
A_{540}=6866496, \quad A_{324}=4732, \quad A_{648}=2548,
\end{gathered}
$$

which are verified by using Matlab.

\section{Conclusion}

Since the weight distributions play an important role in the applications of cyclic codes, this paper focuses on the determination of those for a class of cyclic codes with three nonzeros. Relevant results received a lot of attention by using methods with lower moments of exponential sums. Here we try to apply higher moments to deal with the problem.

\section{Conflict of Interests}

The authors declare that there is no conflict of interests regarding the publication of this paper.

\section{Acknowledgments}

This work was supported in part by the National Basic Research Program of China under Grants 2013CB338004 and 2012 CB316100 and the National Natural Science Foundation of China under Grant 61271222.

\section{References}

[1] D. R. Anderson, "A new class of cyclic codes," SIAM Journal on Applied Mathematics, vol. 16, pp. 181-197, 1968.

[2] F. J. Macwilliams, "On binary cyclic codes which are also cyclic over $G_{2^{s}}$," SIAM Journal on Applied Mathematics, vol. 19, no. 1, pp. 75-95, 1970.

[3] T. Kasami, "A decoding procedure for multiple-error-correcting cyclic codes," IEEE Transactions on Information Theory, vol. 10, pp. 134-138, 1964.

[4] V. Pless and W. C. Huffman, Handbook of Coding Theory, Elsevier, Amsterdam, The Netherlands, 1998.

[5] S. G. S. Shiva and K. C. Fung, "Permutation decoding on certain triple error-correcting binary codes," IEEE Transactions on Information Theory, vol. 18, no. 3, pp. 444-446, 1972.

[6] S. G. S. Shiva, K. C. Fung, and H. S. Y. Tan, "On permutation decoding of binary cyclic double-error-correcting codes of certain lengths," IEEE Transactions on Information Theory, vol. 16, pp. 641-643, 1970.

[7] F. MacWilliams and J. Seery, "The weight distributions of some minimal cyclic codes," IEEE Transactions on Information Theory, vol. 27, no. 6, pp. 796-806, 1981.

[8] R. J. McEliece and J. Rumsey, "Euler products, cyclotomy, and coding," Journal of Number Theory, vol. 4, pp. 302-311, 1972.

[9] M. van der Vlugt, "Hasse-Davenport curves, Gauss sums, and weight distributions of irreducible cyclic codes," Journal of Number Theory, vol. 55, no. 2, pp. 145-159, 1995. 
[10] C. Ding, "The weight distribution of some irreducible cyclic codes," IEEE Transactions on Information Theory, vol. 55, no. 3, pp. 955-960, 2009.

[11] C. Ding and J. Yang, "Hamming weights in irreducible cyclic codes," Discrete Mathematics, vol. 313, no. 4, pp. 434-446, 2013.

[12] R. W. Fitzgerald and J. L. Yucas, "Sums of Gauss sums and weights of irreducible codes," Finite Fields and their Applications, vol. 11, no. 1, pp. 89-110, 2005.

[13] R. J. McEliece, "Irreducible cyclic codes and Gauss sums," in Combinatorics, Part 1: Theory of Designs, Finite Geometry and Coding Theory, vol. 55, pp. 179-196, Mathematica Centrum, Amsterdam, The Netherlands, 1974.

[14] C. Ding, Y. Liu, C. Ma, and L. Zeng, "The weight distributions of the duals of cyclic codes with two zeros," IEEE Transactions on Information Theory, vol. 57, no. 12, pp. 8000-8006, 2011.

[15] K. Feng and J. Luo, "Weight distribution of some reducible cyclic codes," Finite Fields and their Applications, vol. 14, no. 2, pp. 390409, 2008.

[16] J. Luo, Y. Tang, and H. Wang, "Cyclic codes and sequences: the generalized Kasami case," IEEE Transactions on Information Theory, vol. 56, no. 5, pp. 2130-2142, 2010.

[17] M. van der Vlugt, "Surfaces and the weight distribution of a family of codes," IEEE Transactions on Information Theory, vol. 43, no. 4, pp. 1354-1360, 1997.

[18] S. X. Li, S. H. Hu, T. Feng, and G. Ge, "The weight distribution of a class of cyclic codes related to Hermitian forms graphs," IEEE Transactions on Information Theory, vol. 59, no. 5, pp. 30643067, 2013.

[19] X. Liu and Y. Luo, "The weight distributions of some cyclic codes with three or four nonzeros over $\mathbb{F}_{3}$," Designs, Codes and Cryptography, 2013.

[20] M. Xiong, "The weight distributions of a class of cyclic codes II," Designs, Codes and Cryptography, vol. 72, no. 3, pp. 511-528, 2014.

[21] Z. Zhou, C. Ding, J. Luo, and A. Zhang, "A family of five-weight cyclic codes and their weight enumerators," IEEE Transactions on Information Theory, vol. 59, no. 10, pp. 6674-6682, 2013.

[22] A. Johansen and T. Helleseth, "A family of $m$-sequences with five-valued cross correlation," IEEE Transactions on Information Theory, vol. 55, no. 2, pp. 880-887, 2009.

[23] A. Johansen, T. Helleseth, and A. Kholosha, "Further results on $m$-sequences with five-valued cross correlation," IEEE Transactions on Information Theory, vol. 55, no. 12, pp. 5792-5802, 2009.

[24] N. Boston and G. McGuire, "The weight distributions of cyclic codes with two zeros and zeta functions," Journal of Symbolic Computation, vol. 45, no. 7, pp. 723-733, 2010.

[25] R. Lidl and H. Niederreiter, Finite Fields, Cambridge University Press, Cambridge, UK, 1997.

[26] X. Liu and Y. Luo, "On the bounds and achievability about the ODPC of $\mathscr{G} \mathscr{R} \mathscr{M}(2, m)^{*}$ over prime field for increasing message length," Designs, Codes and Cryptography, 2013.

[27] Y. Chen and A. J. H. Vinck, "A lower bound on the optimum distance profiles of the second-order Reed-Muller codes," IEEE Transactions on Information Theory, vol. 56, no. 9, pp. 43094320, 2010.

[28] W. Bosma, J. Cannon, and C. Playoust, "The Magma algebra system. I. The user language," Journal of Symbolic Computation, vol. 24, no. 3-4, pp. 235-265, 1997.

[29] R. Hartshorne, Algebraic Geometry, GTM 52, Springer, New York, NY, USA, 1977.
[30] F. J. MacWilliams and N. J. A. Sloane, The Theory of ErrorCorrecting Codes, North-Holland, Amsterdam, The Netherlands, 1988. 


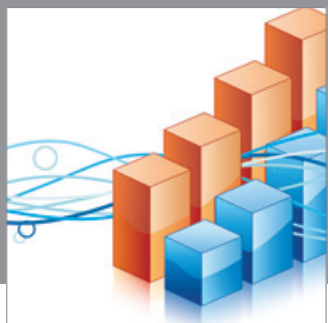

Advances in

Operations Research

mansans

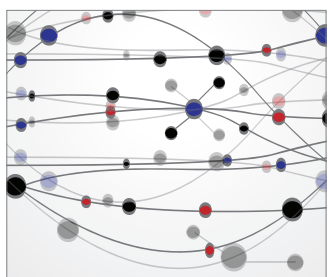

The Scientific World Journal
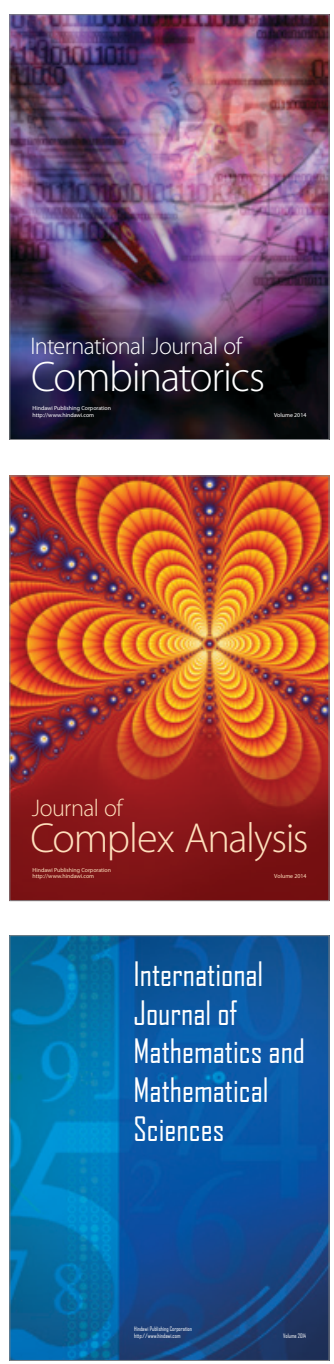
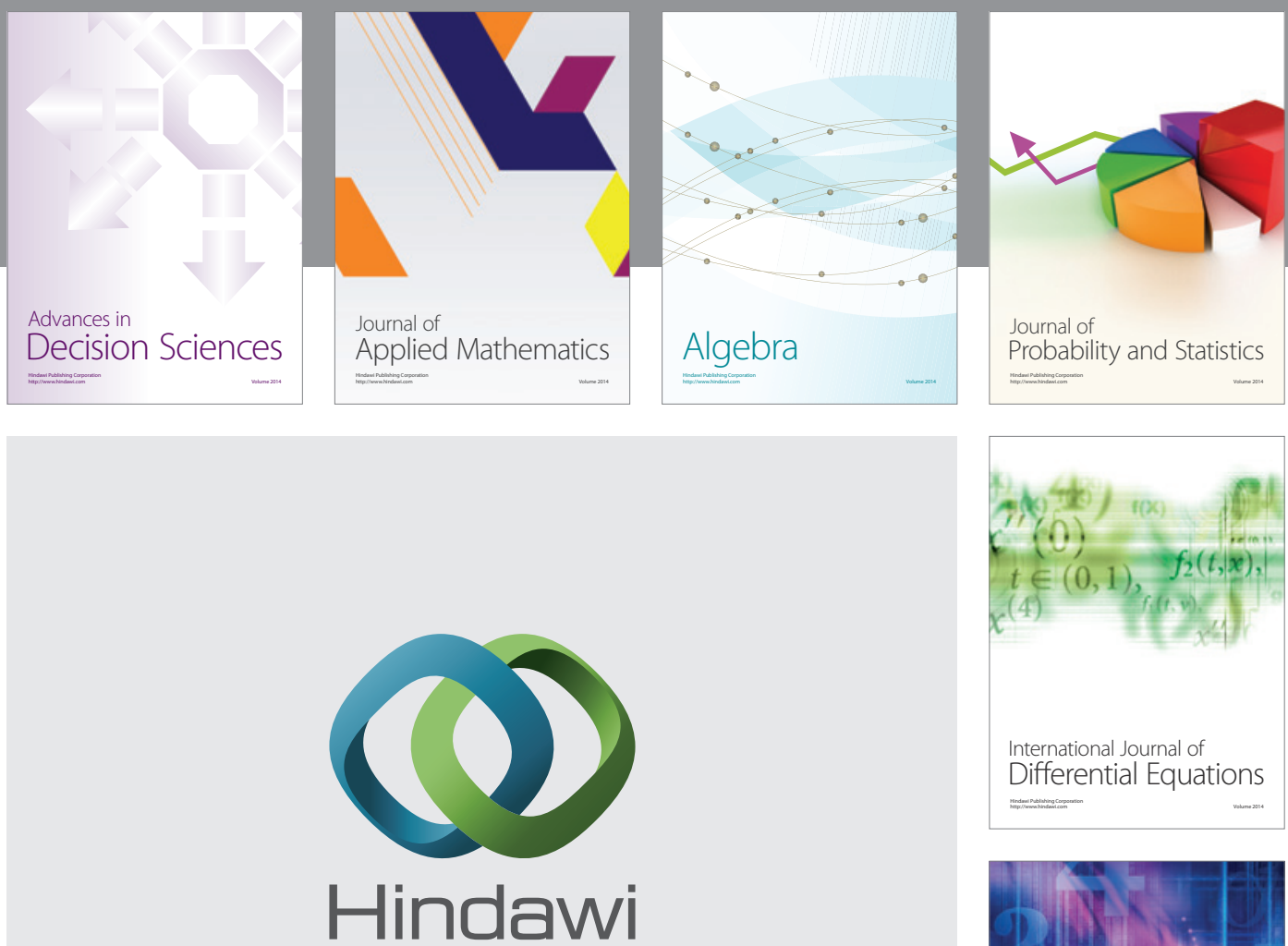

Submit your manuscripts at http://www.hindawi.com
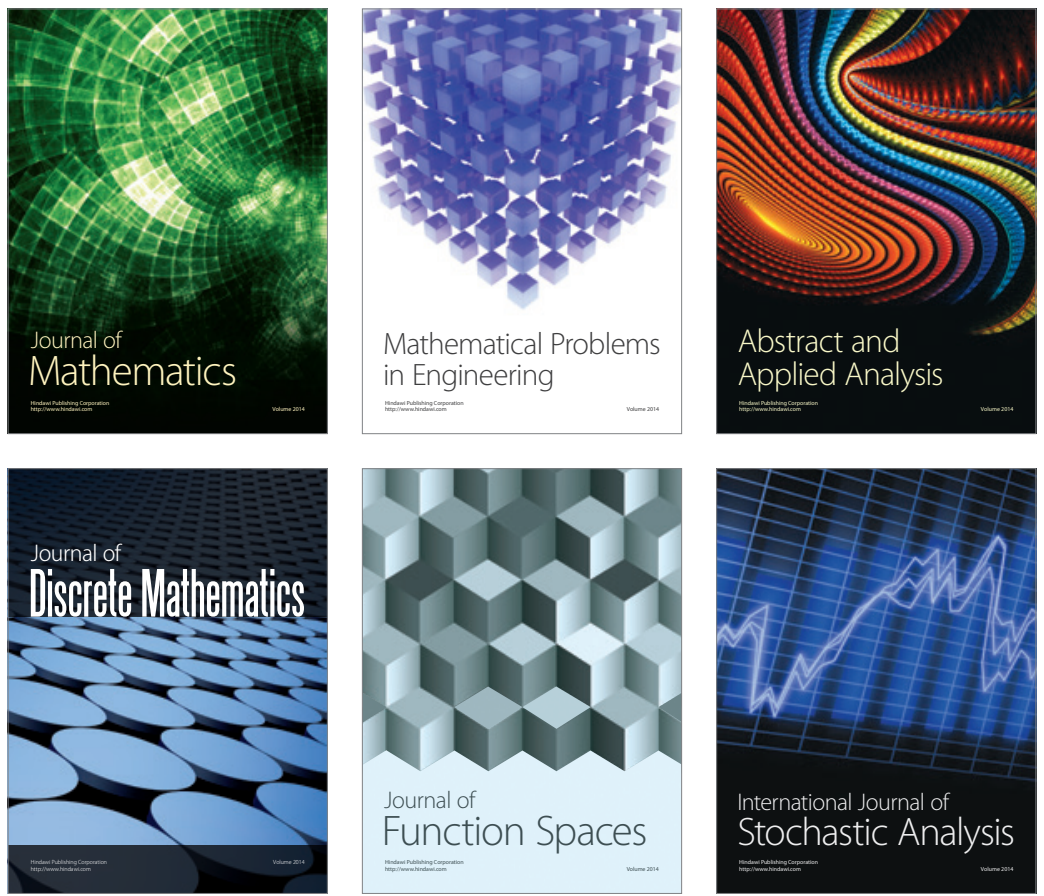

Journal of

Function Spaces

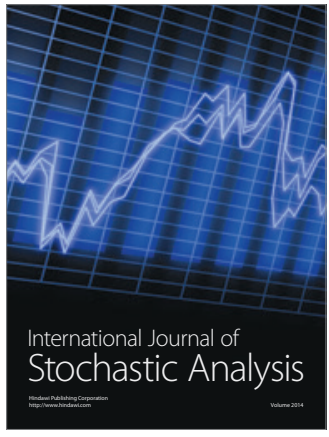

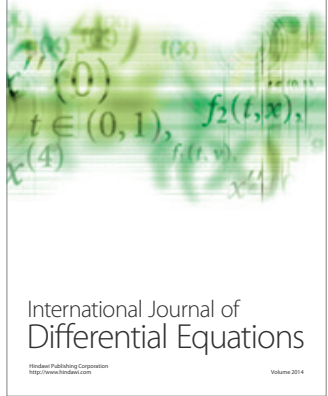
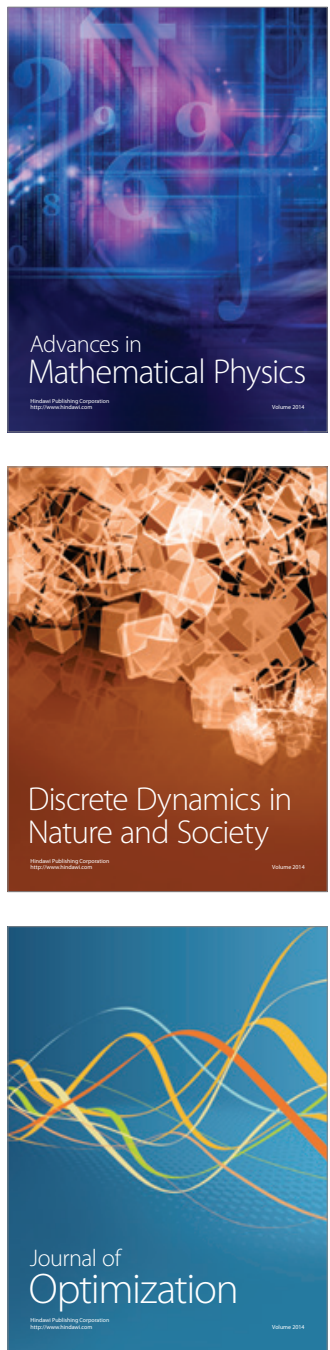